\title{
Values and preferences for hepatitis $C$ self-testing among the general population and healthcare workers in Rwanda
}

\author{
Janvier Serumondo ${ }^{1}$, Sonjelle Shilton ${ }^{2 *}$, Ladislas Nshimiyimana' ${ }^{1}$ Prosper Karame ${ }^{1}$, Donatha Dushimiyimana', \\ Emmanuel Fajardo², Eric Remera' , Gallican N. Rwibasira ${ }^{1}$ and Guillermo Z. Martínez-Pérez ${ }^{2,3}$
}

\begin{abstract}
Background: In 2018, Rwanda launched a 5-year hepatitis C virus (HCV) elimination plan as per the World Health Organization global targets to eliminate HCV by 2030. To improve awareness of HCV status, strategies are needed to ensure easy access to HCV testing by as-yet unreached populations. HCV-self-testing, an innovative strategy, could further increase HCV testing uptake. This assessment explores perceptions around HCV self-testing among members of the public and healthcare workers in Rwanda.
\end{abstract}

Methods: A qualitative study was undertaken in Masaka District Hospital, comprising individual interviews, group interviews and participatory action research (PAR) activities. Purposive and snowball sampling methods guided the selection of informants. Informed consent was obtained from all participants. A thematic analysis approach was used to analyse the findings.

Results: The participants comprised 36 members of the public and 36 healthcare workers. Informants appreciated HCV self-testing as an innovative means of increasing access to HCV testing, as well as an opportunity to test privately and subsequently autonomously decide whether to seek further HCV care. Informants further highlighted the need to make HCV self-testing services free of charge at the nearest health facility. Disadvantages identified included the lack of pre/post-test counselling, as well as the potential psychosocial harm which may result from the use of HCV self-testing.

Conclusion: HCV self-testing is perceived to be an acceptable method to increase HCV testing in Rwanda. Further research is needed to assess the impact of HCV self-testing on HCV cascade of care outcomes.

Keywords: Rwanda, HCV self-test, Screening, Qualitative

\section{Background}

Hepatitis $\mathrm{C}$ virus (HCV) infection results in more than 290,000 deaths a year, with an estimated 58 million people living with chronic hepatitis $C$; however, just $21 \%$ of these cases are diagnosed. Globally 9.4 million people have been treated for $\mathrm{HCV}$ with the majority

*Correspondence: sonjelle.shilton@finddx.org

2 Foundation for Innovative New Diagnostics (FIND), Geneva, Switzerland

Full list of author information is available at the end of the article of those treated in the Eastern Mediterranean Region [1]. The World Health Organization (WHO) recommends focused screening of the most affected populations and of the general population in settings where $\mathrm{HCV}$ prevalence is $\geq 2-5 \%$; all $\mathrm{HCV}$-infected individuals should be treated with pan-genotypic direct-acting antivirals (DAAs) [2-4]. Although focused screening can be performed using rapid diagnostic tests (RDTs), many HCV-infected people in hard-to-reach settings in countries with a high burden of $\mathrm{HCV}$ remain 
undiagnosed. Thus, new strategies are needed to increase $\mathrm{HCV}$ testing coverage in these settings.

As of 2020, more than 28 countries in Africa have $\mathrm{HCV}$ national policies [1]. Rwanda is one of those counties. In Rwanda, the $\mathrm{HCV}$ seroprevalence is $4-5 \%$, with a higher prevalence in people living with HIV (PLHIV) (4.7\%), prisoners (6.5\%), and adults aged $>55$ years (16.5\%) [5]. In December 2018, the Rwandan government launched a 5-year HCV elimination plan that aimed to screen $>4$ million individuals and treat all confirmed cases [5]. The plan involved a phased approach, first targeting high-risk groups, followed by mass screening campaigns using RDTs at the community and health facility level to promote the uptake of HCV testing. To enable last-mile service delivery for elimination, innovative testing approaches, such as HCV self-testing, may be needed.

The usability and acceptability of HIV self-testing as an innovative tool to increase HIV testing uptake and awareness of HIV infection has previously been demonstrated [5-8]. The extensive experience with HIV self-testing is relevant and applicable to $\mathrm{HCV}$ and suggests that HCV self-testing could increase uptake of HCV testing services [6, 9].

As was the case with HIV self-testing prior to its endorsement by WHO in 2016 [8], the values and preferences of relevant populations in relation to $\mathrm{HCV}$ self-testing as an alternative to facility-based $\mathrm{HCV}$ testing must be explored. While evidence about how communities view HCV self-testing remains limited, research from Kyrgyzstan, the United Kingdom and Viet Nam suggests HCV self-testing is widely viewed as acceptable by people who inject drugs (PWID) [1012]. Further evidence on how the general population perceive $\mathrm{HCV}$ self-testing is needed. Healthcare workers' perceptions on HCV self-testing are also important. On one hand, healthcare workers must educate the general public on how to correctly use HCV selftesting and what to do if the result of an HCV self-testing is reactive. On the other hand, healthcare workers are also at occupational risk of $\mathrm{HCV}$ acquisition and, although they are routinely screened for $\mathrm{HCV}$ in many countries, they could also be end-users of HCV selftesting. Here, we describe a qualitative study, conducted in Rwanda, that aimed to assess the values and preferences around HCV self-testing among the general population and healthcare service providers. The findings of this research will help inform the future implementation of $\mathrm{HCV}$ self-testing in Rwanda and neighbouring settings with similar socio-cultural characteristics and a high burden of $\mathrm{HCV}$.

\section{Methods}

\section{Study design and site}

This was a descriptive, cross-sectional, qualitative research study that involved individual interviews, group interviews and participatory action research (PAR) as data collection methods. It was conducted in Masaka District Hospital, Kigali, Rwanda, between August and November 2020. Masaka District Hospital was selected as a suitable site since this is a site where the Rwanda Biomedical Centre (RBC) has conducted $\mathrm{HCV}$-related research, training and patient support activities and where staff has received ongoing education and mentoring on $\mathrm{HCV}$ diagnosis and care.

\section{Population and recruitment}

The study population comprised members of the public and healthcare workers (HCWs). Inclusion criteria for both populations were: being a Rwandan citizen, aged $\geq 18$ years, and willing to give informed consent. Additional inclusion criteria for HCWs were that they were working in the private or public healthcare sector at the time of data collection and actively engaged in $\mathrm{HCV}$ diagnosis and/or care provision services.

Four research team members (two females, two males) recruited informants using a mix of purposive and snowball sampling. For the HCWs, the first seeds were selected from an existing database of physicians and nurses trained by the RBC in HCV care services management.

These HCWs were contacted directly via telephone; they subsequently identified other potential $\mathrm{HCW}$ informants and the first seeds for the public group. These public seeds then recommended other potential informants that met the inclusion criteria.

All potential informants received a telephone call and the study objectives were explained to them. If interested, they were invited to Masaka District Hospital to participate in either an individual or group discussion or in a PAR session. All invited individuals provided written informed consent prior to their enrolment; none declined to participate.

\section{Data collection}

Data collection was conducted face-to-face and using the Kinyarwanda language. Individual and group interviews and PAR sessions were led by the designated team leader (Rwandese, male) from the Rwanda Biomedical Centre. Individual and group interviews were guided by a 42 -item semi-structured guide that aimed to explore participants' knowledge of $\mathrm{HCV}$ and $\mathrm{HCV}$ testing, views around HCV self-testing and preferences for HCV self-testing delivery (Additional file 1). The 
interviews were audio-recorded, transcribed verbatim and translated into English into a single question-byquestion matrix created in MS Excel ${ }^{\circledR}$.

The PAR sessions, which were conducted once all the interviews had been completed, used a set of four exercises to explore participants' preferences for $\mathrm{HCV}$ self-testing delivery (Fig. 1). During the sessions, photographs were taken of the attendees' written and pictorial exercises. All photographs were collated in a single MS Word ${ }^{\circledR}$ document, and their content was transcribed verbatim and translated into English.

\section{Data analysis}

Data collection and analysis were conducted contemporaneously. Thematic analysis, informed by Kielmann and colleagues' guidance for non-social scientists conducting qualitative health research [13], was used to analyse and report the research findings. A pre-defined coding tree was used to deductively code the data in MS Word and in Dedoose software. The coding tree included a series of themes and sub-themes that followed the structure of the data collection instruments. Coding was done in parallel with memoing and with report writing. Analysis was done with a focus on the three main pre-defined themes (i.e., knowledge of $\mathrm{HCV}$ and $\mathrm{HCV}$ testing, views around $\mathrm{HCV}$ self-testing and preferences for HCV selftesting delivery) and comparing informants' narratives by gender, by population group, and by data collection technique. As the analysis started while data collection was ongoing, the preliminary findings from the individual interviews could be discussed during the group interviews, and the preliminary findings from the group interviews could be discussed during the PAR sessions.

\section{Trustworthiness}

Various methods were used to ensure the credibility of the study findings. Triangulation was applied to the choice of study population (maximum variation sampling in age, sex and geographical location of residence), data collection methods (individual and group interviews and PAR), and data analysis approaches, i.e. by the research team, external advisers, and seven Rwanda Biomedical Centre members and partners, who comprised the Technical Working Group, in a final triangulation meeting. Upon finalisation of each individual and group interview, the interviewers jointly analysed the transcripts to reflect on bias that might have affected data collection (i.e., observant, social desirability, memory bias) and topics that merited further exploration in subsequent data generation encounters. During the reporting, emphasis was placed on constant comparison and ensuring that deviant voices were noted.

\section{Ethical considerations}

As part of the informed consent process, all informants were informed about the study aim, the organisations involved, the risks associated with their participation, their right to cease participating at any time, their right to not answer questions they did not want to, and data privacy and confidentiality measures. All informants signed two copies of the consent forms and received one copy themselves. The consent forms were the only documents where the informants' full names were included. No personal identifiers from any audio recording or photograph were transcribed. To prevent any involuntary identification of participants, all recordings were deleted once the analysis was complete. Ethics approval was obtained from the Rwanda National Ethics Committee (Ref. 650/RNEC/2020).

\section{Results \\ Participant characteristics}

The participants comprised 36 members of the public (average age 32 years; 17 females) and $36 \mathrm{HCWs}$ (average age 37 years; 21 females) (Table 1 ). The majority of participants $(n=50)$ lived in urban areas. The most prevalent educational level was a higher education diploma $(\mathrm{n}=30)$, followed by secondary education $(n=19)$ and a bachelor's degree $(n=16)$. Regarding occupation, nurses were the most represented group $(\mathrm{n}=31)$, followed by self-employed individuals $(\mathrm{n}=8)$, students $(n=7)$, and other forms of employment, such as precarious day-to-day jobs $(n=6)$. Most HCWs were recruited from primary healthcare centres $(n=31)$.

\section{Public perceptions around HCV and HCV testing}

Most of the members of the public described HCV as a liver disease that manifested as a distended abdomen. The majority stated that HCV was highly transmissible through blood contact by sharing sharp implements, such as razors and needles; horizontal transmission; working in nursing care; and through unprotected sexual intercourse. The potential for $\mathrm{HCV}$ transmission via oral fluids and close contact such as bed- and clothes-sharing with an $\mathrm{HCV}$-infected person was mentioned. A few informants did not know how $\mathrm{HCV}$ was transmitted.

We know that a liver disease called HCV is transmitted through blood-to-blood contact. A person can be infected by HCV when that person's blood gets in contact with an HCV-infected person's blood. HCV can also be transmitted through sexual intercourse.

(male, 38 years old, information and communication technology (ICT) professional) 


\section{ROLES \& RESPONSIBILITIES}

In this exercise, each group debated:

i) Responsibilities: duties and rights they -as a specific key population- perceived that they have as members of the society, and duties and rights that they perceive that society assigns to them,

ii) main routine tasks or activities that they commonly engage in, and

iii) resources they have access to (financial means, education certificates, housing, etc.).

After having listed all these aspects in a flip chart, the subgroups debated how all these characteristics may put them at increased risk of $\mathrm{HCV}$ or at increased risk of lacking access to HCV care. They debated if there are differences between women's and men's allocation of responsibilities, engagement in tasks, access to and use of resources, and risks to HCV infection. They also compared themselves with the general population or with other key population.

\section{PAR Exercise A}

\section{DECISION-TAKING}

In this exercise, the groups thought, considering the results of exercises $\mathrm{A}$ and $\mathrm{B}$, about any institutional or healthcare system adjustments, capacities, and solutions that would be needed to attend to:

i) their key population's needs to stay HCV-negative and aware of their HCV status,

ii) their obstacles to access HCV prevention, diagnosis and care,

iii) the inequalities that make them fail to use HCV testing services in equal conditions to the general population.

The groups debated the differences between women's and men's needs, obstacles and inequalities in relation to HCV care access, use and benefit. They also compared themselves with the general population.

PAR Exercise C

\section{MAPPING}

In this exercise, each group reflected on some of the roles, tasks and risks identified in Exercise A, and represented them graphically in a map of their own community, village, area, or region that each group drew in a flipchart, with the aim to decide: How HCV testing services could be proposed? In which locations? Run by whom? At what time of the day? Each group decided what to draw.

After drawing the map, the groups thought about the places that members of their key population frequent, their daily activities, their movements around the place, the ways to contact their social, sexual or drug-sharing networks, and on the timing of such movements and activities. The groups could use arrows, clocks, text, or anything they wanted to represent their insights into their map.

\section{PAR Exercise B}

\section{PILING UP}

In this exercise, each group received a set of cards with terms that related to attributes of interest for HCVST delivery:

1) Distribution of HCVST: Door-to-door, vending machine, internet, pharmacy, nurse's office, doctor's office...

2) Cost of HCVST: $0 \$, 2 \$, 5 \$, 10 \$, 25 \$, 50 \$ .$.

3) Specimen type: Blood, saliva, urine.

4) Support: HCVST done alone, with a partner, with a nurse, with a doctor...

5) Instructions: In a leaflet, video tutorial, website after scanning a barcode, provided verbally by an HCW...

6) Reading results: Alone, by phone, in person, in a website...

7) Counselling: In person, hotline, email, with a peer...

8) Linkage to confirmatory testing: Over the telephone, in the nearest clinic, via a local CSO..

The groups piled up each group of cards up according to their own preferences, and thinking in four scenarios: the most and the less preferred option for self-testing for women \& the most and the less preferred option for self-testing for men.

\section{PAR Exercise D}

Fig. 1 A description of the participatory action research (PAR) exercises 
Table 1 Aggregate demographic data for the study informants

\begin{tabular}{|c|c|c|c|c|}
\hline \multirow[t]{3}{*}{ Variable } & \multicolumn{2}{|c|}{$\begin{array}{l}\text { Members of the } \\
\text { public }(n=36)\end{array}$} & \multicolumn{2}{|c|}{$\begin{array}{l}\text { Healthcare } \\
\text { workers }(n=36)\end{array}$} \\
\hline & Females & Males & Females & Males \\
\hline & $n=17$ & $n=19$ & $n=21$ & $\mathrm{n}=15$ \\
\hline \multicolumn{5}{|l|}{ Age group } \\
\hline $18-25$ & 3 & 5 & & \\
\hline $26-33$ & 9 & 2 & & 3 \\
\hline $34-41$ & 3 & 5 & 12 & 8 \\
\hline $42-49$ & & 1 & 4 & 4 \\
\hline $50-57$ & 2 & 3 & 4 & \\
\hline $58-65$ & & 3 & 1 & \\
\hline \multicolumn{5}{|l|}{ Education } \\
\hline Primary & 2 & 3 & & \\
\hline Secondary & 9 & 7 & 3 & \\
\hline Vocational training & 1 & & & \\
\hline Higher (diploma) & 4 & 4 & 13 & 9 \\
\hline Higher (bachelor's degree) & 1 & 5 & 4 & 6 \\
\hline Postgraduate & & & 1 & \\
\hline \multicolumn{5}{|l|}{ Occupation } \\
\hline Farmer & 1 & 1 & & \\
\hline Self-employed & 6 & 2 & & \\
\hline Government employee & & 2 & & \\
\hline Student & 3 & 4 & & \\
\hline Technician & 1 & 3 & & \\
\hline Pastor & & 3 & & \\
\hline Unemployed & 3 & 1 & & \\
\hline Other (survival jobs) & 3 & 3 & & \\
\hline Nurse & & & 19 & 12 \\
\hline Medical doctor & & & 1 & 3 \\
\hline Midwife & & & 1 & \\
\hline \multicolumn{5}{|l|}{$\begin{array}{l}\text { For healthcare workers: type of } \\
\text { facility }\end{array}$} \\
\hline Primary health centre & & & 19 & 12 \\
\hline Hospital & & & 2 & 3 \\
\hline \multicolumn{5}{|l|}{ Setting } \\
\hline Rural & 3 & 8 & 5 & 6 \\
\hline Urban & 14 & 11 & 16 & 9 \\
\hline
\end{tabular}

It was highlighted that older people, generally aged 50 years or more, are at high risk of HCV. Female sex workers and their clients, prison inmates, and blood transfusion recipients were mentioned by a few informants as groups at risk of $\mathrm{HCV}$ infection. HCWs were also identified as people at increased risk of $\mathrm{HCV}$.

I think everyone should get a test so that they can know if they have hepatitis B or hepatitis C. People who are at risk of getting this disease are those working in health settings, as they meet so many patients. (male, 31 years old, farmer)
Diverse opinions were expressed about when and where an individual should be tested for HCV. Some informants suggested that, to increase testing among students, HCV testing events should be conducted during academic holidays. Others suggested that systematic $\mathrm{HCV}$ testing for people nearing their 50s should be organised. However, many informants mentioned that access to the test should be available on-demand, should any person express the need for it. It was known that $\mathrm{HCV}$ testing is available at health centres, although most of the members of the public informants did not have any previous experience of $\mathrm{HCV}$ testing. Just two informants stated they had been tested during a mass screening campaign.

Regarding barriers to accessing $\mathrm{HCV}$ testing, the majority identified the lack of community-level information about $\mathrm{HCV}$, "negligence" about seeking medical check-ups, and financial constraints. It was also added that people are afraid of $\mathrm{HCV}$ testing because of the stigma attached to it due to the community's perception of HCV as highly transmissible and fatal. Some informants noted there may be a lack of time due to conflicting work schedules, the inconvenient testing hours at health facilities, and the geographical inaccessibility of health facilities for some rural inhabitants.

\section{Sometimes people have limited information on $\mathrm{HCV}$. Then they are scared of getting an HCV test, saying that: 'if ever find out I am infected, while that dis- ease is not curable and is severely fatal, then I may have fear about seeking HCV testing.' (male, 21 years old, student)}

\section{HCW's perceptions around HCV and HCV testing}

All nurses expressed that HCV is commonly known as a liver disease and emphasised its silent progression as it remains asymptomatic during its early stages. Progressive tiredness, abdominal distension with ascites, eye and hand jaundice, and leg oedema were mentioned as signs of advanced-stage HCV. They also highlighted that HCV is curable, especially when treatment is initiated at an early stage before the disease has progressed to cirrhosis and liver cancer. The nurses considered those who share sharp implements (e.g. PWID), handle sharp implements (including HCWs) or engage in unprotected sex (e.g. sex workers and their clients) to be groups at increased risk of $\mathrm{HCV}$.

A few nurses recalled that kunywana, a traditional loyalty blood pact that involves mixing and drinking one another's blood, is a practice that puts people at risk of $\mathrm{HCV}$, especially the elderly, because many old people, according to the $\mathrm{HCW}$ informants, may have been exposed to kunywana at some point. 
It's likely found in those at high risk of sharing needles, such as old people, who used to share those needles and injured their bodies through the cultural blood pact kunywana. Again, medical staff is at risk due to permanent exposure to blood or other biological fluids that can contaminate them.

(female, 34 years old, nurse)

HCWs had different views on the circumstances under which HCV testing should be required. It was mentioned that individuals aged 15 years or more should be tested, as adolescents were reported to engage in unprotected sex. Sex workers, PLHIV, married couples, people who share sharp implements, and HCV-infected individuals' family members were also mentioned as groups that should receive $\mathrm{HCV}$ testing.

Unlike the members of the public, most HCWs had previously been tested for $\mathrm{HCV}$, either through routine facility-based $\mathrm{HCV}$ testing or HCW-specific systematic screening for $\mathrm{HCV}$. One physician disclosed how, thanks to routine screening, he discovered his $\mathrm{HCV}$-positive status:

I tested positive. I was lucky. I took medicines and I went for a check-up after ending the treatment and found out that I was HCV-free.

(male, 45 years old, hospital medical doctor)

Similar to the members of the public, all HCWs expressed that $\mathrm{HCV}$ testing is available countrywide and free of charge in hospitals and health centres. Some explained that the Rwanda Biomedical Centre had attempted to increase awareness of $\mathrm{HCV}$ testing using radio advertisements, billboards, and through sensitisation during mass campaigns.

For anyone who wants to get tested for HCV, all health facilities in the country currently provide those services free of charge starting from health centres up to the referral hospitals.

(male, 39 years old, health centre nurse)

HCWs identified different barriers to $\mathrm{HCV}$ testing from those identified by the members of the public. A lack of information on $\mathrm{HCV}$, geographical barriers, and financial constraints were among the most important. In addition, some HCWs declared that people do not demand HCV testing because of the long queues and the time that would be spent at health facilities.

Some HCWs did not identify any barriers to HCV testing as testing services are, allegedly, available and free of charge countrywide. However, in their opinion, some individuals refuse testing because they think that $\mathrm{HCV}$ is incurable. It was also suggested that because viral hepatitis services are integrated with HIV care, some individuals may feel that they are viewed by others as having HIV rather than HCV. In their opinion, the integration of HIV and HCV services might have become a deterrent to $\mathrm{HCV}$ testing.

Due to the merging of a place where HCV test and $H I V$ test are performed, people think that someone who would see them there, would think that it is not for hepatitis, but for HIV.

(female, 37 years old, health centre nurse)

\section{Public perception of usability and acceptability of HCV self-testing}

The informants were not familiar with self-testing for $\mathrm{HCV}$ at home. When asked about their experiences of HCV self-tests, they became confused with other tests that are commonly performed in health settings, such as rapid HCV tests. Most knew self-testing kits are available for diabetes, HIV, malaria, pregnancy, and body temperature. Females mentioned that they were familiar with pregnancy self-tests. Young and middle-aged informants were more familiar with self-testing than older informants.

Privacy and confidentiality were highlighted as advantages of HCV self-testing. Most supported the introduction of HCV self-testing in the community as an opportunity to reduce waiting times at health facilities and allow individuals to know their HCV status. The general population, people aged $\geq 12$ years, couples and elderly people were mentioned as groups that may be interested in HCV self-testing.

A few members of the public highlighted the potential risk of "mental illnesses" for users who received an HCVpositive self-test having not received pre-test counselling. They also mentioned that individuals may test positive and not communicate this to their HCWs.

If you test yourself and find that you are sick, you may have depression. Or, if you have another disease, it can easily get complicated with that liver disease. When it is done from health facilities, they offer counselling before and after the test and they provide treatment immediately. When you do it alone, you may experience a deep depression after knowing that you have the disease.

(female, 28 years old, unemployed)

\section{HCW's perception of usability and acceptability of HCV self-testing}

Most HCWs expressed that HCV self-testing may help users save time, increase work-productivity and reduce the cost of transport to health facilities. Some HCWs underlined that $\mathrm{HCV}$ self-testing would reduce the 
workload at health facilities and increase $\mathrm{HCV}$ testing uptake. Consequently, HCV self-testing could contribute to early treatment initiation and reduce transmission. Most HCWs did not identify any disadvantages in $\mathrm{HCV}$ self-testing. However, in agreement with the members of the public, the risk of non-disclosure of an $\mathrm{HCV}$-positive self-test result, due to fear of stigma, was also highlighted by some.

The only issue would be the follow-up. People may get their results and just be scared of sharing. This means more instructions should be provided so that in case a client self-tests and has an issue, is willing to share the result as the aim is to know the HCV status and get further support for care and treatment.

(female, 43 years old, health centre nurse)

The HCWs identified female sex workers, men who have sex with men (MSM), and HCWs as potential users of HCV self-testing. Some noted that the youth engage in risky behaviours, such as unprotected sex, and lack healthcare-seeking behaviours and would therefore be interested in HCV self-testing.

I think young people will be the ones to enjoy it [HCV self-testing] because they are mostly not interested in receiving healthcare services at the health facility. (female, 34 years old, health centre nurse)

\section{Public preferences for $\mathrm{HCV}$ self-testing service delivery} Most members of the public emphasised that HCV selftests should be available at health centres and pharmacies. This was followed by allowing community health workers to distribute $\mathrm{HCV}$ self-tests, while the tests could also be delivered at health posts. A few participants disagreed with making the kits available in kiosks, as they could be inappropriately stored, leading to false results. The majority preferred services to be available at any time.

I think it would be good if the tests are in pharmacies because most of the time, they would be closer to the community. Someone could easily go there and buy the test at a low price. It could be good also if they would put them in health settings, but it would be good to ensure they are accessible and well stored. (female, 27 years old, student)

On the usability of HCV self-tests, informants emphasised that tests designed for saliva specimens would be the easiest to use, followed by urine specimens. A few mentioned that they would have more trust in tests that used blood specimens. Regarding trustworthiness, most informants said they would trust the results if there were clear instructions on how to use the test correctly. Many added that the acceptance and trustworthiness of the results may be an obstacle to further confirmatory testing and treatment.

...If it is validated that with either blood, saliva or urine, self-testing can get accurate and trustworthy results, the best self-testing methods would be oral and urine, compared to blood.

(male, 38 years old, ICT professional)

The members of the public highlighted the need for any HCV self-test to have clear, easy-to-read and -understand instructions. A common suggestion was that instructions should be visual (e.g. text and pictures showing a step-bystep process). The need to support people who are illiterate or who have visual or cognitive disabilities was raised, to prevent their exclusion from the innovation. Although most informants would prefer to self-test themselves, the majority also highlighted that support from an HCW or via a free hotline would be required.

There is a cheap way in villages through community health workers, then they should provide training to some people and those trained should train/provide demonstrations to the rest of the community. Another option is through the internet because many people are using the internet, I mean using advertisements. Those are the two options I can choose. (male, 31 years old, farmer)

Perceived barriers to HCV self-testing included the lack of information on its availability, unavailability due to stock-outs, and inadequate or hard-to-read instructions. In terms of barriers to accessing HCV diagnosis and treatment, financial constraints were mentioned, particularly if HCV self-testing were expensive, but also due to the possible geographical inaccessibility of the health setting.

During the piling up exercise in the PAR session, members of the public could not reach a consensus regarding a preferred option for the delivery of $\mathrm{HCV}$ self-testing (Fig. 1). Females and males exhibited considerable differences in their preferences for some attributes of $\mathrm{HCV}$ self-testing. In general, most attendees believed that males would prefer to receive HCV self-tests at their home (door-to-door), while females would prefer a variety of settings. Most females and a few males suggested $\mathrm{HCV}$ self-test delivery should be free of charge or at cost in the range of 0.5 to 2 USD. Almost all subgroups in the PAR preferred saliva specimens. Similarly, most subgroups thought that the best strategy for counselling support would be via a free-of-charge hotline. Overall, it was suggested that HCV self-testing would be best if performed and interpreted by the client alone, without 
requiring assistance. Most participants would prefer to receive an explanation of the instructions from a nurse or pharmacist. Almost all groups chose to request confirmatory testing directly from their nearest clinic, but males preferred the linkage via a telephone call.

\section{HCW's preferences for HCV self-testing service delivery}

Overall, HCWs expressed a positive attitude towards $\mathrm{HCV}$ self-testing. The majority emphasised the need to raise awareness about the availability of HCV self-testing and to decentralise HCV self-testing to the lowest level of the health system or make it available in pharmacies at any time. Some preferred HCV self-testing to be delivered by community health workers.

The majority of HCWs preferred the use of non-invasive procedures for HCV self-testing because, in their opinion, some people would fear finger-pricks or other procedures that involved sharp implements. It was noted that specimens such as saliva and urine are easy to collect, although saliva samples were the preferred option. The majority thought that there would be no problem regarding the trustworthiness of the results, as long as the testing procedure was explained to the user.

Using an oral specimen, it is easy, and not a painful method; accepting it would not be a problem.

(male, 45 years old, hospital medical doctor)

Most HCWs agreed that people should perform $\mathrm{HCV}$ self-testing themselves and suggested that HCV self-test providers should explain their use to clients. If someone were to have a problem while using the test, there must be a provider at the health facility or a free hotline to offer support. The need for facility-based counselling for those who received a positive self-test result was highlighted. Some HCWs thought that it would be easier for men to accept a positive result than women, while some people might be "downhearted".

Most informants recognised that illiterate or elderly people might face problems using $\mathrm{HCV}$ self-testing. Problems could occur if users had limited knowledge about the use of HCV self-testing and received a poor explanation from the provider about the use and about what to do following a reactive result. Most HCWs noted that although people would be willing to use $\mathrm{HCV}$ self-tests, they may be unable to access them due to stock-outs or a lack of time, depending on how they are distributed. Other problems included limited access due to high costs.

Difficulties could be related to the time required to get where the HCV self-tests would be sold. The long travel distance and the required time to go where the services would be provided, but also going to the hos- pital or where the HCV self-tests would be availed with the queue, without having any health problem, would be difficult.

(male, 34 years old, health centre nurse)

Some differences between male and female HCWs attending the PAR session were noted for some attributes. HCWs thought that males would prefer to obtain $\mathrm{HCV}$ self-testing kits from vending machines. For females, HCWs thought that vending machines, nurses' or doctors' offices, and pharmacies would be viable options. This high degree of variation in preferred distribution options was also observed among the informants in the members of the public group. All informants suggested delivery of HCV self-tests should be free of charge. Almost all subgroups preferred saliva specimens, with no difference between female and male subgroups. Nearly all groups agreed that HCV self-testing should be performed and the result interpreted by the user alone without assistance. This option was reportedly more preferable for males than females. Post-test counselling options varied from peer educator to in-person, with females preferring more in-person counselling support. Most subgroups favoured the availability of a confirmatory test upon receiving a positive self-test result. Most attendees to the PAR session preferred instructions to be given by the vendor or an HCV self-test provider. Almost all attendees opined thatthe best strategy for linkageto-care after a positive HCV self-test result would be to instruct end-users to request immediate confirmatory testing at their nearest clinic.

\section{Discussion}

This research shows that HCV self-testing is viewed by members of the public and HCWs alike to be an acceptable approach to HCV testing, which could increase individuals' HCV status awareness and become a useful tool to help Rwanda meet its HCV elimination targets. For $\mathrm{HCV}$ self-testing to have maximum impact, improved awareness and knowledge around $\mathrm{HCV}$ among the Rwandan population is needed. As in other HCV selftesting acceptability studies, among MSM and PWID in Viet Nam [11] and among PWID in London [10], members of the Rwandese public and HCWs expressed positive attitudes towards HCV self-testing.

We believe that our informants' attitudes towards $\mathrm{HCV}$ self-testing might have been influenced by their existing knowledge of self-testing devices (e.g. for HIV and malaria); their perception that minimal social harm would occur as a consequence of HCV self-testing; and their perception that HCV self-testing can be performed alone without any assistance. For all informants, potential advantages of $\mathrm{HCV}$ self-testing included potential 
increases in the number of people tested for HCV and earlier treatment initiation. For HCWs, they specifically noted that reductions in queues and workloads at health facilities could also be among the positive impacts of HCV self-testing. These perceived benefits around selftesting have previously been identified in HIV self-testing programme evaluations, which also found that HIV self-testing may increase HIV testing uptake and accelerate access to antiretroviral treatment $[14,15]$. There is considerable research evidence from various East African countries to suggest that positive attitudes exist towards the use of HIV self-testing [16-21]. Based on this, it is reasonable to think that the acceptability seen in our study could also be seen in future HCV self-testing acceptability studies carried out in the region [10-12].

Pre-delivery acceptability may not correlate with acceptability of HCV self-testing during its delivery to the Rwandese population if their preferences for access and use are not considered. For HCV self-testing to be acceptable after its endorsement by national regulatory authorities, our informants stressed the need for decentralised delivery models. HCV self-tests should be available, at any time, in pharmacies, health centres and health posts, as well as from community health workers. The informants also highlighted the need to offer confirmatory tests in the nearest clinic as the favoured strategy for linkage-to-care following a positive $\mathrm{HCV}$ self-test. Notably, a recent systematic review reported that decentralised HCV self-testing service delivery models that include task-shifting and integration of $\mathrm{HCV}$ care in primary healthcare could help ensure that HCV self-test users have accelerated paths linking them to confirmatory testing and initiation of HCV treatment [22].

Almost all informants considered that obtaining saliva specimens would be easier and less painful than obtaining blood and would be preferable for people who fear finger-pricks or any procedure involving sharp implements. This preference for oral HCV self-tests was also reported by PWID in London [10], male young offenders in England [23], and Vietnamese MSM and PWID [11]. This finding is also aligned with a review of factors enabling HIV self-testing uptake, which highlighted a global preference for oral HIV self-tests [24].

Our informants also discussed willingness-to-pay for an $\mathrm{HCV}$ self-test. Most informants expressed a preference for $\mathrm{HCV}$ self-tests to be provided free to the most underserved groups and that, were a cost to the end-user unavoidable, it should be between 0.5 and 2.0 USD. This preference is noteworthy, as sustainability is a concern for many health authorities in low- and middle-income countries, such as Rwanda. The fact that some people in Rwanda may be willing to bear a minimum cost for a diagnostic device provided that it is made freely available to the most underserved individuals merits further exploration prior to the deployment of HCV self-tests in the country.

PLHIV, prisoners, and female sex workers and their clients were identified as among the most underserved groups that would merit special consideration due to their, allegedly, increased risk of HCV acquisition. The nurses in the present study opined that those who share and handle sharp implements and those who engage in unprotected sex could benefit from HCV self-testing. This was a reflection that drew on risky practices rather than sexual, disease or occupational identities. HCV selftest programmers must adopt a similar non-stigmatising approach to avoid creating additional discrimination against already marginalised groups. Irrespective of their personal characteristics, individuals should be educated about which specific practices (sexual or otherwise) may put them at increased risk of contracting HCV, HIV and other blood-borne infections. Such education can help people make informed decisions on which diagnostics (including $\mathrm{HCV}$ self-tests) to use after engaging in risky behaviour and which linkage-to-care they should demand from HCWs (including confirmatory testing following a positive HCV self-test).

In our study, elderly people (a non-stigmatised group in Rwanda) were identified as being a group at risk of $\mathrm{HCV}$. Elders could have been exposed, according to the nurses' narratives, during kunywana, a traditional loyalty blood pact. It is unclear how prevalent kunywana is today, but previous studies have reported a high prevalence of HCV among older people in Rwanda [25, 26]. One study reported that traditional operations or scarification are risky practices that could lead to $\mathrm{HCV}$ acquisition in Rwanda [25]. Irrespective of how prevalent kunywana, or similar traditions, are in Rwanda, this study finding suggests the need for $\mathrm{HCV}$ programme planners to regard the general population holistically, rather than separating the population into non-marginalised and marginalised groups, and take full account of culture-specific practices in any future HCV self-testing education and awareness activities.

Although both members of the public and $\mathrm{HCW}$ informants shared many preferences for the attributes of HCV self-testing implementation, the PAR sessions showed that males might prefer to access HCV self-tests via vending machines or home-delivery options, while females expressed a preference for accessing HCV selftests through their pharmacist, nurse or doctor. These differences may be related to the traditional gendered distribution of roles and tasks between females and males in Rwanda. This distribution might also mean males are more engaged in jobs and social activities with schedules that conflict with those of clinics. It may also be related 
to the fact that females tend to exhibit more healthcare-seeking behaviour and more favourable attitudes to accessing conventional healthcare environments for health education and illness prevention purposes than males.

\section{Challenges for future HCV self-testing}

End-user and healthcare provider preferences for $\mathrm{HCV}$ self-testing must be considered by HCV care programmers, alongside anticipated barriers to HCV self-testing delivery. Financial limitations, poor healthcare-seeking behaviours, and an absence of information about HCV diagnosis were some of the barriers to conventional $\mathrm{HCV}$ testing identified by our informants. It was also noted that in Rwanda, HCV self-testing implementers will need to avoid HCV self-tests becoming unavailable due to stock-outs, ensure end-users do not struggle with hardto-interpret instructions, and be aware that many people may be unable to access HCV self-testing unless provided for free. The HCW informants added that people might also be discouraged by the long waiting times at health facilities, should HCV self-tests be distributed in clinics or should they need to travel to a clinic to obtain confirmatory testing. Some of these barriers could be fuelled if end-users receive inadequate pre-test counselling from HCWs or if the HCV self-test instructions and mechanisms for linkage-to-care are not tailored to include people with reading or cognitive difficulties.

Time constraints and a lack of information were previously reported barriers for hospital-based screening for HCV $[27,28]$. A review of qualitative studies of HIV self-testing also mentioned these factors as barriers to HIV self-testing uptake [24]. Other reported hindrances to HIV self-testing uptake are an inability to purchase the kits, fear of unexpected results, the risk of psychological distress, and perceived inability to understand the kit instructions $[18,21,24,29,30]$.

Lessons from the introduction of HIV self-testing must be considered for the successful implementation of $\mathrm{HCV}$ self-testing. HIV self-testing has increased HIV testing uptake, particularly in East Africa [24, 31-33]. HIV selftesting offers the opportunity to test sexual partners, and it promotes earlier treatment initiation [16, 19, 34]. Compared with conventional facility-based HIV testing, HIV self-testing yields benefits in terms of maintaining privacy, saving time and resources, informing sexual health decision-making, and reducing the anxiety experienced when waiting for results in a conventional HIV testing scenario $[21,29,34]$.

The successful implementation of HIV self-testing was determined by the knowledge around HIV, perceived risk of HIV, maintenance of privacy, and easy access to HIV self-tests $[24,29]$. As expressed by our study informants, improved knowledge around $\mathrm{HCV}$ and $\mathrm{HCV}$ self-testing, awareness of risk behaviours that may lead to $\mathrm{HCV}$ acquisition, and delivery of HCV self-testing as per people's preferences so they can access and use it at their own convenience are also among the factors that may help to successfully deliver $\mathrm{HCV}$ in a safe and effective way in Rwanda.

\section{Limitations and future prospects}

All HCW participants in this study were engaged in HCV care and were aware of the Rwanda Biomedical Centre position in the Rwandan hepatitis response. Hence, social desirability could have influenced their responses. Despite the possibility of social desirability, the HCWs were better positioned than the members of the public to provide insightful opinions about the advantages of $\mathrm{HCV}$ self-testing and the barriers to its delivery in Rwanda. This is not only because of their previous participation in HCV trainings but also because HCW informants had more experience with $\mathrm{HCV}$ testing themselves than the informants who comprised the members of the public group since, in Rwanda, all HCWs should be routinely tested for HCV [35].

A limitation was that sampling and recruitment occurred in a limited geographic space: the Masaka District Hospital and neighbouring primary health care catchment areas. Travelling and social gathering restrictions to halt the spread of the SARS-CoV-2 virus in Rwanda applied at the time of study conduct. Enrolment of informants from remote rural could have improved the richness and diversity of insights on HCV self-testing.

While the findings of this study may characterise the values and preferences for $\mathrm{HCV}$ self-testing of members of the public and HCWs, caution is warranted to not generalise these findings to marginalised groups that, as per the informants' opinions, might be at increased risk of $\mathrm{HCV}$. In Rwanda, groups that are perceived by members of the public and HCWs to be people who engage in unprotected sex and who share contaminated sharp implements may have different values and preferences for HCV self-testing. Further quantitative studies are recommended to explore Rwandese people's preferences for HCV self-testing, paying special attention to the inhabitants of the most remote areas as well as to populations who see their right to $\mathrm{HCV}$ care being neglected.

\section{Conclusion}

This study reports a high level of acceptability of $\mathrm{HCV}$ self-testing among Rwandan members of the public and among HCWs engaged in $\mathrm{HCV}$ diagnosis and care. $\mathrm{HCV}$ self-testing is perceived as an innovative strategy to increase access to $\mathrm{HCV}$ testing and to improve individuals' awareness of their HCV status. Alongside 
increased awareness around the causes of $\mathrm{HCV}$, the distribution of HCV self-tests at the community-level, free of charge to at least the most socio-economically underserved groups, and with clear instructions for use and for linkage to confirmatory testing, would allow the Rwandan population to access HCV self-testing and use it appropriately, at their own convenience. Less privileged groups may have different preferences; therefore, the health authorities should consider a myriad of delivery models in any future implementation of $\mathrm{HCV}$ self-testing in Rwanda.

\section{Abbreviations}

DAA: Direct-acting antiviral; FIND: Foundation for Innovative New Diagnostics; HCV: Hepatitis C virus; HIV: Human immunodeficiency virus; MSM: Men who have sex with men; PAR: Participatory action research; PLHIV: People living with HIV; PWID: People who inject drugs; WHO: World Health Organization.

\section{Supplementary Information}

The online version contains supplementary material available at https://doi. org/10.1186/s12879-021-06773-6.

\section{Additional file 1: Annex 1. Guide for Individual \& Group Interviews}

\section{Acknowledgements}

We would like to thank all of the participants in Rwanda who contributed to the interviews and group sessions. We thank as well the WHO HIV/Hepatitis and STI team for their input on the larger values and preferences assessment of which this study was a part of. Medical writing assistance and editorial support, under the direction of the authors and funded by FIND, was provided by Adam Bodley, according to Good Publication Practice guidelines.

\section{Authors' contributions}

JS: contextualization of the protocol to Rwandan context, data curation, formal analysis, writing- original draft. SS: conceptualization, methodology, supervision, validation, review and editing. LN: data curation, formal analysis. PK: data curation, formal analysis. DD: data curation, formal analysis. EF: review and editing. ER: data curation, formal analysis. GR: data curation, formal analysis. GP: methodology, creation of protocol, validation, support to country team, review and editing. All authors have read and approved the manuscript.

\section{Funding}

This work was part of the HEAD (Hepatitis C Elimination through Access to Diagnostics)-Start project, supported by Unitaid.

\section{Availability of data and materials}

The datasets used and/or analysed during the current study are available from the corresponding author on reasonable request.

\section{Declarations}

\section{Ethics approval and consent to participate}

Ethics approval was obtained from the Rwanda National Ethics Committee (Ref. 650/RNEC/2020). All informants provided written informed consent. All methods were carried out in accordance with relevant guidelines and regulations.

\section{Consent for publication}

Not applicable.

\section{Competing interests}

The authors have no conflicting or competing interests to declare.

\section{Author details}

${ }^{1}$ Rwanda Biomedical Centre (RBC), Kigali, Rwanda. ${ }^{2}$ Foundation for Innovative New Diagnostics (FIND), Geneva, Switzerland. ${ }^{3}$ Department of Physiatrics and Nursing, University of Saragossa, Zaragoza, Spain.

Received: 18 June 2021 Accepted: 1 October 2021

Published online: 14 October 2021

\section{References}

1. WHO. Global progress report on HIV, viral hepatitis and sexually transmitted infections. Geneva: WHO; 2021.

2. Pawlotsky JM. New hepatitis $C$ therapies: the toolbox, strategies, and challenges. Gastroenterology. 2014;146(5):1176-92.

3. Feeney ER, Chung RT. Antiviral treatment of hepatitis C. BMJ. 2014;348:3308.

4. WHO. Guidelines on hepatitis B and C testing. Geneva: WHO; 2017.

5. Umutesi G, Shumbusho F, Kateera F, Serumondo J, Kabahizi J, Musabeyezu $\mathrm{E}$, et al. Rwanda launches a 5-year national hepatitis C elimination plan: a landmark in sub-Saharan Africa. J Hepatol. 2019;70(6):1043-5.

6. Riou J, Aït Ahmed M, Blake A, Vozlinsky S, Brichler S, Eholié S, et al. Hepatitis $C$ virus seroprevalence in adults in Africa: a systematic review and meta-analysis. J Viral Hepat. 2016:23(4):244-55.

7. WHO. Guidelines on HIV self-testing and partner notification - supplement to consolidated guidelines on HIV testing services. Geneva: WHO; 2016.

8. Calderon Y, Cowan E, Schramm C, Stern S, Brusalis C, Iscoe M, et al. HCV and HBV testing acceptability and knowledge among urban emergency department patients and pharmacy clients. Prev Med. 2014;61:29-33.

9. Zhang C, Li X, Brecht ML, Koniak-Griffin D. Can self-testing increase HIV testing among men who have sex with men: a systematic review and meta-analysis. PLoS ONE. 2017;12(11):e0188890.

10. Guise A, Witzel TC, Mandal S, Sabin C, Rhodes T, Nardone A, et al. A qualitative assessment of the acceptability of hepatitis $C$ remote self-testing and self-sampling amongst people who use drugs in London, UK. BMC Infect Dis. 2018;18(1):281.

11. Nguyen LT, Nguyen VTT, Le Ai KA, Truong MB, Tran TTM, Jamil MS, et al. Acceptability and usability of HCV self-testing in high risk populations in Vietnam. Diagnostics (Basel). 2021;11(2):1.

12. Martínez-Pérez GZ, Nikitinb DS, Bessonovac A, Fajardod E, Bessonovc S, Shilton S. Values and preferences for hepatitis $C$ self-testing among people who inject drugs in Kyrgyzstan

13. Kielmann K, Cataldo F. Introduction to qualitative research methodology. London: DFID; 2011.

14. Figueroa C, Johnson C, Ford N, Sands A, Dalal S, Meurant R, et al. Reliability of HIV rapid diagnostic tests for self-testing compared with testing by health-care workers: a systematic review and meta-analysis. Lancet HIV. 2018;5(6):e277-90.

15. Tucker JD, Wei C, Pendse R, Lo YR. HIV self-testing among key populations: an implementation science approach to evaluating self-testing. J Virus Erad. 2015;1(1):38-42.

16. Matovu JK, Buregyeya E, Arinaitwe J, Wanyenze RK.'... if you bring the kit home, you [can] get time and test together with your partner": Pregnant women and male partners' perceptions regarding female partnerdelivered HIV self-testing in Uganda - a qualitative study.' Int J STD AIDS. 2017;28(13):1341-7.

17. Izizag BB, Situakibanza H, Mbutiwi T, Ingwe R, Kiazayawoko F, Nkodila A, et al. Factors associated with acceptability of HIV self-testing (HIVST) among university students in a Peri-Urban area of the Democratic Republic of Congo (DRC). Pan Afr Med J. 2018;31:248.

18. Peck RB, Lim JM, van Rooyen H, Mukoma W, Chepuka L, Bansil P, et al. What should the ideal HIV self-test look like? A usability study of test prototypes in unsupervised HIV self-testing in Kenya, Malawi, and South Africa. AIDS Behav. 2014;18(Suppl 4):S422-32.

19. Kalibala S, Tun W, Cherutich P, Nganga A, Oweya E, Oluoch P. Factors associated with acceptability of HIV self-testing among health care workers in Kenya. AIDS Behav. 2014;18:S405-14.

20. Kurth AE, Cleland CM, Chhun N, Sidle JE, Were E, Naanyu V, et al. Accuracy and acceptability of oral fluid HIV self-testing in a general adult population in Kenya. AIDS Behav. 2016;20(4):870-9. 
21. Dzinamarira T, Muvunyi CM, Kamanzi C, Mashamba-Thompson TP. HIV self-testing in Rwanda: awareness and acceptability among male clinic attendees in Kigali, Rwanda: a cross-sectional survey. Heliyon. 2020;6(3):e03515.

22. Oru E, Trickey A, Shirali R, Kanters S, Easterbrook P. Decentralisation, integration, and task-shifting in hepatitis $C$ virus infection testing and treatment: a global systematic review and meta-analysis. Lancet Glob Health. 2021;9(4):e431-45.

23. Candfield S, Samuel MI, Ritchie D, McDonald C, Brady M, Taylor C. Use and acceptability of salivary hepatitis $C$ virus testing in an English Young Offender Institution. Int J STD AIDS. 2017;28(12):1234-8.

24. Njau B, Covin C, Lisasi E, Damian D, Mushi D, Boulle A, et al. A systematic review of qualitative evidence on factors enabling and deterring uptake of HIV self-testing in Africa. BMC Public Health. 2019;19(1):1289.

25. Makuza JD, Liu CY, Ntihabose CK, Dushimiyimana D, Umuraza S, Nisingizwe MP, et al. Risk factors for viral hepatitis $C$ infection in Rwanda: results from a nationwide screening program. BMC Infect Dis. 2019;19(1):688.

26. Umumararungu E, Ntaganda F, Kagira J, Maina N. Prevalence of hepatitis $C$ virus infection and its risk factors among patients attending Rwanda Military Hospital, Rwanda. Biomed Res Int. 2017:2017:5841272.

27. Shehata N, Austin T, Ha S, Timmerman K. Barriers to and facilitators of hepatitis $C$ virus screening and testing: a scoping review. Can Commun Dis Rep. 2018;44(7-8):166-72.

28. Ju FB, Dumpe M, Lane GB, Shanks L. Awareness, compliance, and barriers of 1-time hepatitis $C$ screening in hospital-based providers. J Nurse Pract. 2020;16:837.

29. Ngure K, Heffron R, Mugo N, Thomson KA, Irungu E, Njuguna N, et al. Feasibility and acceptability of HIV self-testing among pre-exposure prophylaxis users in Kenya. J Int AIDS Soc. 2017;20(1):21234
30. Bwana P, Ochieng L, Mwau M. Performance and usability evaluation of the INSTI HIV self-test in Kenya for qualitative detection of antibodies to HIV. PLoS ONE. 2018;13(9):e0202491.

31. Njau B, Damian DJ, Abdullahi L, Boulle A, Mathews C. The effects of HIV self-testing on the uptake of HIV testing, linkage to antiretroviral treatment and social harms among adults in Africa: a systematic review and meta-analysis. PLoS ONE. 2021;16(1):e0245498.

32. Eshun-Wilson I, Jamil MS, Glidden DV, Cheryl J, Noelle T, et al. A systematic review and network meta-analyses to assess the effectiveness of HIV selftesting distribution strategies. Clin Infect Dis. 2021;73(4):e1018-28.

33. Johnson CC, Kennedy C, Fonner V, Siegfried N, Figueroa C, Dalal S, et al. Examining the effects of HIV self-testing compared to standard HIV testing services: a systematic review and meta-analysis. J Int AIDS Soc. 2017;20(1):21594.

34. Hamilton A, Thompson N, Choko AT, Hlongwa M, Jolly P, Korte JE, et al. HIV self-testing uptake and intervention strategies among men in subSaharan Africa: a systematic review. Front Public Health. 2021;9:594298.

35. Mbituyumuremyi A, Van Nuil Jl, Umuhire J, Mugabo J, Mwumvaneza M, Makuza JD, et al. Controlling hepatitis C in Rwanda: a framework for a national response. Bull World Health Organ. 2018;96(1):51-8.

\section{Publisher's Note}

Springer Nature remains neutral with regard to jurisdictional claims in published maps and institutional affiliations.
Ready to submit your research? Choose BMC and benefit from:

- fast, convenient online submission

- thorough peer review by experienced researchers in your field

- rapid publication on acceptance

- support for research data, including large and complex data types

- gold Open Access which fosters wider collaboration and increased citations

- maximum visibility for your research: over $100 \mathrm{M}$ website views per year

At BMC, research is always in progress.

Learn more biomedcentral.com/submissions 